\title{
A METHOD OF ESTABLISHING THE 27-LINE CONFIGURATION ON A CUBIC SURFACE
}

\author{
By W. P. MiLne.
}

[Received September 13th, 1911.-Revised October 23rd, 1911. - Read November 9th, 1911.]

1. The present communication gives a method whereby, when a certain homographic correspondence has been established, the configuration and chief fundamental properties of the 27 lines on a cubic surface become almost intuitive. The method was suggested by, and is the extension of, a method published in the Quarterly Joumal, No. 167, 1911, in which I gave an elementary proof of Salmon's theorem in connexion with the cross-ratio of the four tangents to a cubic curve drawn from a point on itself. Thereafter I used the method to prove the chief focal and bitangent properties of circular cubics and bicircular quartics in two pepers published in the Proceedings of the Edinburgh Mathematical Society, Session 1910-1911. The present paper exhibits many of the properties of the 27 lines on the cubic surface as extensions of corresponding properties in the plene cubic.

2. If we assume that the existence of one line on the cubic surface has been established, it is easy to see that any plane containing this line cuts the surface besides in a conic and that, for five positions of this plane, the conic degenerates into two straight lines. We shall hereafter refer to these five planes as the five tangent planes drawn from this line to the surface.

Let $A B$ be a line on the surface, $B C$ and $C A$ two other lines on the surface meeting $A B$. Let $D A B$ be one (other than $C A B$ ) of the five planes through $A B$ containing degenerate conics of section, and let $D$ be the intersection of the two lines on the surface lying in this latter plane. The cubic surface then assumes the form

$$
a x^{2} t+b y^{2} t+c z^{2} t+2 d t^{2} z+2 f y z t+2 g z x t+2 h x y t+2 k x y z=0
$$

if we take $A B C D$ as the tetrahedron of reference. 
Let $t=\lambda x$ be a plane passing through the line $B C$ and cutting the surface again in the conic $S$. Then the equation to the cone $D S$ is

$$
a x^{2}+b y^{2}+c z^{2}+2\left(f+\frac{k}{\lambda}\right) y z+2(g+d \lambda) z x+2 h x y=0 .
$$

Similarly, if the plane $t=\mu y$ through $C A$ cut the surface again in the conic $S^{\prime}$, the equation to the cone $D S^{\prime}$ is

$$
a x^{2}+b y^{2}+c z^{2}+2(f+d \mu) y z+2\left(g+\frac{k}{\mu}\right) z x+2 h x y=0 .
$$

Thus the two cones $D S$ and $D S^{\prime}$ will be identical if

$$
\lambda \mu=\frac{k}{d} .
$$

Thus, if a plane be drawn through $B C$ and if its conic of section $S$ be joined to $D$, the second degree cone $D S$ cuts the cubic surface in a curve of the sixth degree consisting of $S$, the two lines on the surface through $D$ and another conic $S^{\prime}$ whose plane passes through $C A$.

$A$ one-to-one algebraic correspondence exists between the above two planes containing $S$ and $S^{\prime}$ respectively in virtue of (4).

Any one ray of the cone $D S$ (other than the lines on the surface through $D$ ) is sufficient to identify the plane containing $S^{\prime}$ and thus to find corresponding members of the homography.

3. If $S$ breaks up into two lines, it is plain that $S^{\prime}$ will also consist of two straight lines, and thus the four planes through $B C$ containing degenerate conics correspond to the four planes through $C A$ containing degenerate conics. Furthermore, the line joining the point of intersection of the two lines composing $S$ to $D$ plainly passes through the meeting point of the two lines composing $S^{\prime}$, and a similar property holds whatever point $D$ we choose of the four points of contact of the planes containing degenerate conics which can be drawn through $A B$. We thus have the following theorems:-

The three groups of four tangent planes (other than the plane containing the three given lines on the surface) that can be drawn from three coplanar lines on the surface have equal cross-ratios.

The points of contact constitute the vertices of three "desmic tetrahedra." 
If $a$ and $b$ be two coplanar lines on the surface, the cross-ratio of the four planes joining a to the points of contact of the four tangent planes from $b$ is equal to the cross-ratio of the four planes joining $b$ to the points of contact of the four tangent planes from $a$.

For, let $\alpha, \beta$ be the points of contact of representative tangent planes from $a, b$ respectively. Then the line $\alpha \beta$ passes through one or other of the points of contact of tangent planes from $c$, where $c$ is the line on the surface coplanar with $a$ and $b$. Hence, by Art. $2, a \beta$ and $b a$ are corresponding planes according to one of the four homographic systems defined by the points of contact of the four tangent planes from $c$. The theorem is then evident.

4. The geometrical configuration of the 27 lines, and in particular the intersection diagram of Mr. G. T. Bennett, given in the Proc. London Math. Soc., Series 2, Vol. 9, Part 5, can now be quite easily verified. For example, in terms of the usual notation let $a_{1}, b_{2}, c_{12}$ be three coplanar lines of the system, and let $a_{2}, b_{1}$ be two other coplanar lines of the system meeting $c_{12}$. Consider $a_{3}, a_{4}, a_{5}, a_{6}$. The planes $b_{1} a_{8}, b_{1} a_{4}, b_{1} a_{5}$, $b_{1} a_{6}$ meet the surface again in $c_{13}, c_{14}, c_{15}, c_{16}$ which are thus determined. The lines meeting $a_{1}$, complementary to $c_{13}, c_{14}, c_{15}, c_{16}$, are respectively $b_{3}, b_{4}, b_{5}, b_{6}$, which joined to $a_{2}$ give $c_{23}, c_{24}, c_{25}, c_{26}$ respectively. Thus all the lines meeting $a_{1}$ and $b_{2}$ are arranged as far as $a_{2}$ and $b_{1}$ are concerned. Similarly, we can arrange all the lines meeting $a_{1}$ and $b_{2}$ with reference to another pair of coplanar lines meeting $c_{12}$.

It is found, also, that the correspondences of the special places which give line-pairs occur with double exchanges of these line-pairs. We proceed to tabulate these exchanges. Let the tangent planes from $a_{1}$ be $a_{1}, a_{2}, a_{3}, a_{4}$, and let their corresponding planes with respect to one of the points of contact of the four tangent planes from $b_{2}$ be $\beta_{1}, \beta_{2}, \beta_{3}, \beta_{4}$ as explained in Art. 2. Denoting the four homographies thus obtained by I, II, III, IV respectively, we obtain the following sets of corresponding planes:-

$$
\begin{aligned}
& {\left[\alpha_{1} \alpha_{2} \alpha_{3} \alpha_{4}\right] \equiv\left[\beta_{1} \beta_{2} \beta_{3} \beta_{4}\right],} \\
& {\left[\alpha_{1} \alpha_{2} \alpha_{3} \alpha_{4}\right] \equiv\left[\beta_{2} \beta_{1} \beta_{4} \beta_{3}\right],} \\
& {\left[\alpha_{1} \alpha_{2} \alpha_{3} \alpha_{4}\right] \equiv\left[\beta_{3} \beta_{4} \beta_{1} \beta_{2}\right],} \\
& {\left[\alpha_{1} \alpha_{2} \alpha_{3} \alpha_{4}\right] \equiv\left[\beta_{4} \beta_{3} \beta_{2} \beta_{1}\right] .}
\end{aligned}
$$


Let

\begin{tabular}{|c|c|c|c|}
\hline & atair & $\theta$ & $b_{3}, c_{18}$ \\
\hline$x_{2}$ & ", & $"$ & $b_{4}, c_{14}$ \\
\hline$\alpha_{8}$ & $"$ & $"$ & $b_{5}, c_{15}$ \\
\hline$\alpha_{4}$ & $"$ & $"$ & $b_{6}, c_{10}$ \\
\hline$\beta_{1}$ & $"$ & $"$ & $a_{3}, c_{2}$ \\
\hline$\beta_{2}$ & $"$ & $"$ & \\
\hline$\beta_{3}$ & $"$ & , & \\
\hline$\beta_{4}$ & " & & \\
\hline
\end{tabular}

Next, let $a b$ denote the plane containing the lines $a, b$, and let us rewrite the above correspondences (I), (II), (III), (IV) in terms of the lines lying in the various planes, with the proviso that lines in the same vertical column are coplanar. We thus obtain the following :-

I.

$\begin{array}{lllll}b_{3} c_{13}\left(a_{1}\right) & b_{4} c_{14}\left(\alpha_{2}\right) & b_{5} & c_{15}\left(a_{9}\right) & b_{6} c_{16}\left(a_{4}\right) \\ c_{23} a_{3}\left(\beta_{1}\right) & c_{24} a_{4}\left(\beta_{2}\right) & c_{25} a_{5}\left(\beta_{9}\right) & c_{26} a_{6}\left(\beta_{4}\right) \\ a_{2} b_{1} & a_{2} b_{1} & a_{2} b_{1} & a_{2} b_{1} .\end{array}$

II.

$\begin{array}{llll}b_{3} c_{13}\left(\alpha_{1}\right) & b_{4} c_{14}\left(\alpha_{2}\right) & c_{15} b_{5}\left(\alpha_{3}\right) & c_{16} b_{6}\left(\alpha_{4}\right) \\ a_{4} c_{24}\left(\beta_{2}\right) & a_{3} c_{23}\left(\beta_{1}\right) & c_{26} a_{6}\left(\beta_{4}\right) & c_{25} a_{5}\left(\beta_{9}\right) \\ c_{34} c_{56} & c_{34} c_{56} & c_{34} c_{56} & c_{34} c_{56} .\end{array}$

III.

$\begin{array}{llll}b_{3} c_{13}\left(\alpha_{1}\right) & c_{14} b_{4}\left(\alpha_{2}\right) & b_{5} c_{15}\left(\alpha_{3}\right) & c_{16} b_{6}\left(\alpha_{4}\right) \\ a_{5} c_{25}\left(\beta_{8}\right) & c_{26} a_{6}\left(\beta_{4}\right) & a_{3} c_{29}\left(\beta_{1}\right) & c_{24} a_{4}\left(\beta_{2}\right) \\ c_{35} c_{46} & c_{35} c_{46} & c_{35} c_{46} & c_{35} c_{46} .\end{array}$

IV.

$\begin{array}{llll}b_{3} c_{13}\left(a_{1}\right) & c_{14} b_{4}\left(a_{2}\right) & c_{15} b_{5}\left(a_{3}\right) & b_{6} c_{16}\left(a_{4}\right) \\ a_{6} c_{26}\left(\beta_{4}\right) & c_{25} a_{5}\left(\beta_{3}\right) & c_{24} a_{4}\left(\beta_{2}\right) & a_{3} c_{23}\left(\beta_{1}\right) \\ c_{36} c_{45} & c_{36} c_{45} & c_{36} c_{45} & c_{36} c_{45} .\end{array}$

To explain the above table, we note that the order of the lines $b_{3}, c_{13}$ in the plane $a_{1}$ is kept unaltered throughout. Then, when we make interchanges of the " $\beta$ " planes (e.g., in passing from I to II, $\beta_{1}$ and $\beta_{2}$, $\beta_{3}$ and $\beta_{4}$ ), we interchange the order of the lines in each of $\alpha_{3}$ and $\alpha_{4}$, and also in each of $\beta_{1}$ and $\beta_{2}$. Thus we obtain II from I. Again, in passing from II to III, we interchange $\beta_{1}$ and $\beta_{4}, \beta_{2}$ and $\beta_{3}$. We therefore interchange the lines in $\alpha_{2}$ and $\alpha_{3}, \beta_{1}$ and $\beta_{4}$, and so on.

SER. 2. vor. 10. พo. 1126 . 
5. Some of the other theorems described in Mr. Bennett's paper also follow at once. Thus with the relation $\lambda \mu=$ const., zero and infinity are a pair of corresponding values, and hence the plane $B C A$ through $B C$ corresponds to $C A D$ through $C A$, and $B C D$ through $B C$ corresponds to $C A B$ through $C A$. Interpreting this result in the notation of the double-six, we obtain

$$
a_{1}\left[b_{2} c_{12}, b_{3} c_{13}, b_{4} c_{14}, b_{5} c_{15}, b_{6} c_{16}\right] \equiv b_{2}\left[a_{2} b_{1}, c_{29} a_{3}, c_{24} a_{4}, c_{25} a_{5}, c_{26} a_{6}\right] \text {, }
$$

where $b_{2} c_{12}$ denotes the intersection of the lines $b_{2}$ and $c_{12}$, etc. In this result is contained Dixon's theorem, viz.,

$$
a_{1}\left[b_{3} b_{4} b_{5} b_{6}\right]=b_{2}\left[a_{3} a_{4} a_{5} a_{6}\right]
$$

where $a_{1} b_{3}$ denotes the plane containing $a_{1}$ and $b_{3}$, etc. The various perspectives are also evident, e.g., $a_{1}\left[b_{3} b_{4} b_{5} b_{6}\right]$ joined respectively to $b_{2}\left[c_{23} c_{24} c_{25} c_{26}\right]$ meeting in $a_{2} c_{12}$, where $a_{1} b_{3}$ denotes the intersection of $a_{1}$ and $b_{3}$, etc. 